\title{
Coupled fixed point theorems on complex partial metric space using different type of contractive conditions
}

\author{
M. Gunaseelan, L. N. Mishra
}

\begin{abstract}
In this paper, we obtain coupled fixed point theorems on complex partial metric space using different type of contractive conditions. An example to support our result is presented.
\end{abstract}

Keywords: Coupled fixed point; complex partial metric space.

\section{Introduction}

In many branches of science, economics, computer science, engineering and the development of nonlinear analysis, the fixed point theory is one of the most important tool. In 1989, Backhtin [2] introduced the concept of b-metric space. In 1993, Czerwik [3] extended the results of b-metric spaces. Azam et al.[4] introduced new spaces called complex valued metric spaces and established the existence of fixed point theorems under the contraction condition. P. Dhivya and M. Marudai [5] introduced new spaces called complex partial metric space and established the existence of common fixed point theorems under the contraction condition of rational expression. Bhaskar and Lakshmikantham [7] introduced the concept of coupled fixed point. Ćirić and Lakshmikantham [8] investigated some more coupled fixed point theorems in partially ordered sets. Hassen Aydi [1] introduced coupled fixed point results on partial metric spaces. In this paper, we introduced coupled fixed point results on complex partial metric spaces under the contractive condition.

\section{Preliminaries}

Let $\mathbb{C}$ be the set of complex numbers and $c_{1}, c_{2} \in \mathbb{C}$. Define a partial order $\preceq$ on $\mathbb{C}$ as follows:

Manuscript received July 20, 2019; accepted November 15, 2019.

M. Gunaseelan is with the Department of Mathematics, Sri Sankara Arts and Science College,Enathur, Kanchipuram, India; L. N. Mishra is with the Department of Mathematics, School of Advanced Sciences, Vellore Institute of Technology (VIT) University, Vellore 632 014, Tamil Nadu, India. 
$c_{1} \preceq c_{2}$ if and only if $\operatorname{Re}\left(c_{1}\right) \leq \operatorname{Re}\left(c_{2}\right)$ and $\operatorname{Im}\left(c_{1}\right) \leq \operatorname{Im}\left(c_{2}\right)$.

Consequently,one can infer that $c_{1} \preceq c_{2}$ if one of the following conditions is satisfied:

(i) $\operatorname{Re}\left(c_{1}\right)=\operatorname{Re}\left(c_{2}\right), \operatorname{Im}\left(c_{1}\right)<\operatorname{Im}\left(c_{2}\right)$,

(ii) $\operatorname{Re}\left(c_{1}\right)<\operatorname{Re}\left(c_{2}\right), \operatorname{Im}\left(c_{1}\right)=\operatorname{Im}\left(c_{2}\right)$,

(iii) $\operatorname{Re}\left(c_{1}\right)<\operatorname{Re}\left(c_{2}\right), \operatorname{Im}\left(c_{1}\right)<\operatorname{Im}\left(c_{2}\right)$,

(iv) $\operatorname{Re}\left(c_{1}\right)=\operatorname{Re}\left(c_{2}\right), \operatorname{Im}\left(c_{1}\right)=\operatorname{Im}\left(c_{2}\right)$.

In particular,we write $c_{1} \precsim c_{2}$ if $c_{1} \neq c_{2}$ and one of $(i),(i i)$ and (iii) is satisfied and we write $c_{1} \prec c_{2}$ if only (iii) is satisfied.Notice that

(a) If $0 \preceq c_{1} \precsim c_{2}$,then $\left|c_{1}\right|<\left|c_{2}\right|$,

(b) If $c_{1} \preceq c_{2}$ and $c_{2} \prec c_{3}$ then $c_{1} \prec c_{3}$,

(c) If $a, b \in \mathbb{R}$ and $a \leq b$ then $a c \preceq b c$ for all $c \in \mathbb{C}$.

Definition 2.1. [5] A complex partial metric on a non-empty set $U$ is a function $\xi_{c}: U \times$ $U \rightarrow \mathbb{C}^{+}$such that for all $p, r, s \in Y$ :

(i) $0 \preceq \xi_{c}(p, p) \preceq \xi_{c}(p, r)($ smallself-distances $)$

(ii) $\xi_{c}(p, r)=\xi_{c}(r, p)$ (symmetry)

(iii) $\xi_{c}(p, p)=\xi_{c}(p, r)=\xi_{c}(r, r)$ if and only if $p=r$ (equality)

(iv) $\xi_{c}(p, r) \preceq \xi_{c}(p, s)+\xi_{c}(s, r)-\xi_{c}(s, s)$ (triangularity).

A complex partial metric space is a pair $\left(U, \xi_{c}\right)$ such that $U$ is a non empty set and $\xi_{c}$ is complex partial metric on $U$.

For the complex partial metric $\xi_{c}$ on $U$, the function $d_{\xi_{c}}: U \times U \rightarrow \mathbb{C}^{+}$given by $\xi_{c}^{t}=$ $2 \xi_{c}(p, r)-\xi_{c}(p, p)-\xi_{c}(r, r)$ is a (usual) metric on $U$. Each complex partial metric $\xi_{c}$ on $U$ generates a topology $\tau_{\xi_{c}}$ on $U$ with the base family of open $\xi_{c}$-balls $\left\{B_{\xi_{c}}(p, \varepsilon): p \in U, \varepsilon>\right.$ $0\}$, where $B_{\xi_{c}}(p, \varepsilon)=\left\{r \in U: \xi_{c}(p, r)<\xi_{c}(p, p)+\varepsilon\right\}$ for all $p \in U$ and $0<\varepsilon \in \mathbb{C}^{+}$.

Definition 2.2. [5] Let $\left(U, \xi_{c}\right)$ be a complex partial metric space(CPMS). A sequence $\left(p_{n}\right)$ in a CPMS $\left(U, \xi_{c}\right)$ is converges to $p \in U$, if for every $0 \prec \varepsilon \in \mathbb{C}^{+}$there is $N \in \mathbb{N}$ such that for all $n \in \mathbb{N}$ we get $p_{n} \in B_{\xi_{c}}(p, \varepsilon)$

Definition 2.3. [5] Let $\left(U, \xi_{c}\right)$ be a complex partial metric space. A sequence $\left(p_{n}\right)$ in a CPMS $\left(U, \xi_{c}\right)$ is called Cauchy if there is a $\in \mathbb{C}^{+}$such that for every $\varepsilon \prec 0$ there is $N \in \mathbb{N}$ such that for all $n, m \geq N\left|\xi_{c}\left(p_{n}, p_{m}\right)-a\right|<\varepsilon$.

Definition 2.4. [5] Let $\left(U, \xi_{c}\right)$ be a complex partial metric space(CPMS).

(1) A CPMS $\left(U, \xi_{c}\right)$ is said to be complete if a Cauchy sequence $\left(p_{n}\right)$ in $U$ converges, with respect to $\tau_{\xi_{c}}$, to a point $p \in U$ such that $\xi_{c}(p, p)=\lim _{n, m \rightarrow \infty} \xi_{c}\left(p_{n}, p_{m}\right)$.

(2) A mapping $H: U \rightarrow U$ is said to be continuous at $p_{0} \in U$ if for every $\varepsilon \prec 0$, there exist $\delta>0$ such that $H\left(B_{\xi_{c}}\left(p_{0}, \delta\right)\right) \subset B_{\xi_{c}}\left(H\left(p_{0}, \varepsilon\right)\right)$.

Lemma 2.1. [5] Let $\left(U, \xi_{c}\right)$ be a complex partial metric space. A sequence $\left\{y_{n}\right\}$ is Cauchy sequence in the CPMS $\left(U, \xi_{c}\right)$ then $\left\{y_{n}\right\}$ is Cauchy in a metric space $\left(U, \xi_{c}^{t}\right)$. 
Definition 2.5. Let $\left(U, \xi_{c}\right)$ be a complex partial metric space(CPMS).Then an element $(p, r) \in U \times U$ is said to be a coupled fixed point of the mapping $F: U \times U \rightarrow U$ if $F(p, r)=p$ and $F(r, p)=r$.

Theorem 2.2. Let $\left(U, \xi_{c}\right)$ be a complete complex partial metric space. Suppose that the mapping $\phi: U \times U \rightarrow U$ satisfies the following contractive condition for all $\alpha, \beta, \gamma, \delta \in U$

$$
\xi_{c}(\phi(\alpha, \beta), \phi(\gamma, \delta)) \preceq k \xi_{c}(\phi(\alpha, \beta), \alpha)+l \xi_{c}(\phi(\gamma, \delta), \gamma),
$$

where $k, l$ are nonnegative constants with $k+l<1$. Then, $\phi$ has a unique coupled fixed point.

Proof. Choose $u_{0}, v_{0} \in U$ and set $u_{1}=\phi\left(u_{0}, v_{0}\right)$ and $v_{1}=\phi\left(v_{0}, u_{0}\right)$.Continuing this process, set $u_{n+1}=\phi\left(u_{n}, v_{n}\right)$ and $v_{n+1}=\phi\left(v_{n}, u_{n}\right)$.

Then,

$$
\begin{aligned}
\xi_{c}\left(u_{n}, u_{n+1}\right) & =\xi_{c}\left(\phi\left(u_{n-1}, v_{n-1}\right), \phi\left(u_{n}, v_{n}\right)\right) \\
& \preceq k \xi_{c}\left(\phi\left(u_{n-1}, v_{n-1}\right), u_{n-1}\right)+l \xi_{c}\left(\phi\left(u_{n}, v_{n}\right), u_{n}\right) \\
& =k \xi_{c}\left(u_{n}, u_{n-1}\right)+l \xi_{c}\left(u_{n+1}, u_{n}\right) \\
\xi_{c}\left(u_{n}, u_{n+1}\right) & \preceq \frac{k}{1-l} \xi_{c}\left(u_{n}, u_{n-1}\right)
\end{aligned}
$$

which implies that

$$
\left|\xi_{c}\left(u_{n}, u_{n+1}\right)\right| \leq p\left|\xi_{c}\left(u_{n-1}, u_{n}\right)\right|
$$

where $p=\frac{k}{1-l}<1$. Similarly, one can prove that

$$
\left|\xi_{c}\left(v_{n}, v_{n+1}\right)\right| \leq p\left|\xi\left(v_{n-1}, v_{n}\right)\right|
$$

From (1) and (2), we get

$$
\left|\xi_{c}\left(u_{n}, u_{n+1}\right)\right|+\left|\xi_{c}\left(v_{n}, v_{n+1}\right)\right| \leq p\left(\left|\xi_{c}\left(u_{n-1}, u_{n}\right)\right|+\mid \xi_{c}\left(v_{n-1}, v_{n}\right)\right)
$$

where $p<1$.

Also,

$$
\begin{array}{r}
\left|\xi_{c}\left(u_{n+1}, v_{n+2}\right)\right| \leq p\left|\xi_{c}\left(u_{n}, u_{n+1}\right)\right| \\
\left|\xi_{c}\left(v_{n+1}, v_{n+2}\right)\right| \leq p\left|\xi_{c}\left(v_{n}, v_{n+1}\right)\right|
\end{array}
$$

From (3) and (4), we get

$$
\left|\xi_{c}\left(u_{n+1}, v_{n+2}\right)\right|+\left|\xi_{c}\left(v_{n+1}, v_{n+2}\right)\right| \leq p\left(\left|\xi_{c}\left(u_{n}, u_{n+1}\right)\right|+\left|\xi_{c}\left(v_{n}, v_{n+1}\right)\right|\right)
$$


Repeating this way, we get

$$
\begin{aligned}
\left|\xi_{c}\left(u_{n}, v_{n+1}\right)\right|+\left|\xi_{c}\left(v_{n}, v_{n+1}\right)\right| & \leq p\left(\left|\xi_{c}\left(v_{n-1}, v_{n}\right)\right|+\left|\xi_{c}\left(u_{n-1}, u_{n}\right)\right|\right) \\
& \leq p^{2}\left(\left|\xi_{c}\left(v_{n-2}, v_{n-1}\right)\right|+\left|\xi_{c}\left(u_{n-2}, u_{n-1}\right)\right|\right) \\
& \leq \cdots \leq p^{n}\left(\left|\xi_{c}\left(v_{0}, v_{1}\right)\right|+\left|\xi_{c}\left(u_{0}, u_{1}\right)\right|\right)
\end{aligned}
$$

Now, if $\left|\xi_{c}\left(u_{n}, v_{n+1}\right)\right|+\left|\xi_{c}\left(v_{n}, v_{n+1}\right)\right|=t_{n}$, then

$$
t_{n} \leq p t_{n-1} \leq \cdots \leq p^{n} t_{0}
$$

If $t_{0}=0$ then $\left|\xi_{c}\left(u_{0}, u_{1}\right)\right|+\left|\xi_{c}\left(v_{0}, v_{1}\right)\right|=0$. Hence $u_{0}=u_{1}=\phi\left(u_{0}, v_{0}\right)$ and $v_{0}=v_{1}=$ $\phi\left(v_{0}, v_{0}\right)$, which implies that $\left(u_{0}, v_{0}\right)$ is a coupled fixed point of $\phi$.

Let $t_{0}>0$. For each $n \geq m$, we have

$$
\begin{aligned}
\xi_{c}\left(u_{n}, u_{m}\right) & \preceq \xi_{c}\left(u_{n}, u_{n-1}\right)+\xi_{c}\left(u_{n-1}, u_{n-2}\right)-\xi_{c}\left(u_{n-1}, u_{n-1}\right) \\
& +\xi_{c}\left(u_{n-2}, u_{n-3}\right)+\xi_{c}\left(u_{n-3}, u_{n-4}\right)-\xi_{c}\left(u_{n-3}, u_{n-3}\right) \\
& +\cdots+\xi_{c}\left(u_{m+2}, u_{m+1}\right)+\xi_{c}\left(u_{m+1}, u_{m}\right)-\xi_{c}\left(u_{m+1}, u_{m+1}\right) \\
& \preceq \xi_{c}\left(u_{n}, u_{n-1}\right)+\xi_{c}\left(u_{n-1}, u_{n-2}\right)+\cdots+\xi_{c}\left(u_{m+1}, u_{m}\right)
\end{aligned}
$$

which implies that

$$
\left|\xi_{c}\left(u_{n}, u_{m}\right)\right| \leq\left|\xi_{c}\left(u_{n}, u_{n-1}\right)\right|+\left|\xi_{c}\left(u_{n-1}, u_{n-2}\right)\right|+\cdots+\left|\xi_{c}\left(u_{m+1}, u_{m}\right)\right| .
$$

Similarly,one can prove that

$$
\left|\xi_{c}\left(v_{n}, v_{m}\right)\right| \leq\left|\xi_{c}\left(v_{n}, v_{n-1}\right)\right|+\left|\xi_{c}\left(v_{n-1}, v_{n-2}\right)\right|+\cdots+\left|\xi_{c}\left(v_{m+1}, v_{m}\right)\right| .
$$

Thus,

$$
\begin{aligned}
\left|\xi_{c}\left(u_{n}, u_{m}\right)\right|+\left|\xi_{c}\left(v_{n}, v_{m}\right)\right| & \leq t_{n-1}+t_{n-2}+t_{n-3}+\cdots+t_{m} \\
& \leq\left(p^{n-1}+p^{n-2}+\cdots+p^{m}\right) t_{0} \\
& \leq \frac{p^{m}}{1-p} t_{0} \rightarrow 0 \quad n \rightarrow \infty .
\end{aligned}
$$

which implies that $\left\{u_{n}\right\}$ and $\left\{v_{n}\right\}$ are Cauchy sequence in $\left(U, \xi_{c}\right)$. Since the partial metric space $\left(U, \xi_{c}\right)$ is complete, there exists $u, v \in U$ such that $\left\{u_{n}\right\} \rightarrow u$ and $v_{n} \rightarrow v$ as $n \rightarrow$ $\infty$ and $\xi_{c}(u, u)=\lim _{n \rightarrow \infty} \xi_{c}\left(u, u_{n}\right)=\lim _{n, m \rightarrow \infty} \xi_{c}\left(u_{n}, u_{m}\right)=0, \xi_{c}(u, u)=\lim _{n \rightarrow \infty} \xi_{c}\left(v, v_{n}\right)=$ $\lim _{n, m \rightarrow \infty} \xi_{c}\left(v_{n}, v_{m}\right)=0$. We now show that $u=\phi(p, q)$. We suppose on the contrary that 
$u \neq \phi(u, v)$ and $v \neq \phi(v, u)$ so that $0 \prec \xi_{c}(u, \phi(u, v))=l_{1}$ and $0 \prec \xi_{c}(v, \phi(v, u))=l_{2}$ then

$$
\begin{aligned}
l_{1}=\xi_{c}(u, \phi(u, v)) & \preceq \xi_{c}\left(u, u_{n+1}\right)+\xi_{c}\left(u_{n+1}, \phi(u, v)\right) \\
& =\xi_{c}\left(u, u_{n+1}\right)+\xi_{c}\left(\phi\left(u_{n}, v_{n}\right), \phi(u, v)\right) \\
& \preceq \xi_{c}\left(u, u_{n+1}\right)+k \xi_{c}\left(u_{n-1}, u_{n}\right)+l \xi_{c}(\phi(u, v), u) \\
& \preceq \frac{1}{1-l} \xi_{c}\left(u, u_{n+1}\right)+\frac{k}{1-l} \xi\left(u_{n-1}, u_{n}\right)
\end{aligned}
$$

which implies that

$$
\left|l_{1}\right| \leq \frac{1}{1-l}\left|\xi_{c}\left(u, u_{n+1}\right)\right|+\frac{k}{1-l}\left|\xi\left(u_{n-1}, u_{n}\right)\right|
$$

As $n \rightarrow \infty,\left|l_{1}\right| \leq 0$. Which is a contradiction, therefore $\left|\xi_{c}(u, \phi(u, v))\right|=0$ implies $u=$ $\phi(u, v)$. Similarly we can prove that $v=\phi(v, u)$. Thus $(u, v)$ is a coupled fixed point of $\phi$. Now, if $(g, h)$ is another coupled fixed point of $\phi$, then

$$
\begin{aligned}
\xi_{c}(u, g)=\xi_{c}(\phi(u, v), \phi(g, h)) & \preceq k \xi_{c}(\phi(u, v), u)+l \xi_{c}(\phi(g, h), g) \\
& =k \xi_{c}(u, u)+l \xi_{c}(g, g)=0
\end{aligned}
$$

Thus, we have $g=u$. Similarly, we get $h=v$. Therefore $\phi$ has a unique coupled fixed point

Corollary 2.3. Let $\left(U, \xi_{c}\right)$ be a complete complex partial metric space. Suppose that the mapping $\phi: U \times U \rightarrow U$ satisfies the following contractive condition for all $\alpha, \beta, \gamma, \delta \in U$

$$
\xi_{c}(\phi(\alpha, \beta), \phi(\gamma, \delta)) \preceq \frac{k}{2}\left(\xi_{c}(\phi(\alpha, \beta), \alpha)+\xi_{c}(\phi(\gamma, \delta), \gamma)\right),
$$

where $0 \leq k<1$. Then, $\phi$ has a unique coupled fixed point.

Example 2.4. Let $U=[0, \infty)$ endowed with the usual complex partial metric $\xi_{c}: U \times U \rightarrow$ $[0, \infty)$ defined by $\xi_{c}(p, q)=\max \{p, q\}(1+i)$. The complex partial metric space $\left(U, \xi_{c}\right)$ is complete because $\left(U, \xi_{c}^{t}\right)$ is complete. Indeed,for any $p, q \in U$,

$$
\begin{aligned}
\xi_{c}^{t} & =2 \xi_{c}(p, r)-\xi_{c}(p, p)-\xi_{c}(r, r) \\
& =2 \max \{p, q\}(1+i)-(p+i p)-(q+i q) \\
& =|p-q|+i|p-q| .
\end{aligned}
$$


Thus, $\left(U, \xi_{c}\right)$ is the Euclidean complex metric space which is complete. Consider the mapping $\phi: U \times U \rightarrow U$ defined by $\phi(p, q)=\frac{p+q}{12}$. For any $p, q, g, h \in U$, we have

$$
\begin{aligned}
\xi_{c}(\phi(p, q), \phi(g, h)) & =\frac{1}{12} \max \{p+g, \phi(p, q)+\phi(g, h)\}(1+i) \\
& \leq \frac{1}{12}[\max \{\phi(p, q), p\}+\max \{\phi(g, h), g\}](1+i) \\
& =\frac{1}{12}\left[\xi_{c}(\phi(p, q), p)+\xi_{c}(\phi(g, h), g)\right] .
\end{aligned}
$$

which is the contractive condition (6) for $k=\frac{1}{6}$. Therefore, by Corollary 2.3, and hence $\psi$ has a unique coupled fixed point, which is $(0,0)$. Note that if the mapping $\phi: U \times U \rightarrow U$ is given by $\phi(p, q)=\frac{p+q}{2}$, then $\phi$ satisfies the contractive condition (6) for $k=1$, that is,

$$
\begin{aligned}
\xi_{c}(\phi(p, q), \phi(g, h)) & =\frac{1}{2} \max \{p+g, \phi(p, q)+\phi(g, h)\}(1+i) \\
& \leq[\max \{\phi(p, q), p\}+\max \{\phi(g, h), g\}](1+i) \\
& =\frac{1}{2}\left[\xi_{c}(\phi(p, q), p)+\xi_{c}(\phi(g, h), g)\right] .
\end{aligned}
$$

In this case, $(0,0)$ and $(1,1)$ are both coupled fixed points of $\phi$, and, hence, the coupled fixed point of $\phi$ is not unique. This shows that the condition $k<1$ in Corollary 2.3, and hence $k+l<1$ in Theorem 2.2 cannot be omitted in the statement of the aforesaid results.

\section{References}

[1] H. AYDI, Some Coupled fixed point results on partial metric spaces, International Journal of Mathematics and Mathematics Sciences, Volume 2011, Article ID 647091,11 pages.

[2] I.A.BAKHTIN, The contraction mappings principle in quasi-metric spaces, Functional Analysis, vol.30,pp.26-37,1989(Russian).

[3] S.CZERwick, Contraction mappings in b-metric spaces, Acta Mathematica et Informatica Universitatis Ostraviensis, 1 (1993) 5-11.

[4] A. AZAM B. Fisher AND M. KHAN, Common fixed point theorems in complex valued metric spaces ,Numerical Functional Analysis and Optimization 32(3),(2011) 243-253.

[5] P.DHIVyA, M. MARUdAI, Common fixed point theorems for mappings satisfying a contractive condition of rational expression on a ordered complex partial metric space, Cogent Mathematics(2017),4:1389622.

[6] I. Altun, F. Sola And H. Simsek, Generalized contractions on partial metric spaces, Topology and Its Applications, 157 (18) (2010) 2778-2785.

[7] T. Gnana Bhaskar, V. Lakshmikantham, Fixed point theorems in partially ordered metric spaces and applications, Nonlinear Analysis: Theory, Methods and Applications, 65 (7) (2006) 1379-1393. 
[8] LJ. ĆIRIĆ, V. LAKSHMIKANTHAM, Coupled fixed point theorems for nonlinear contractions in partially ordered metric spaces and applications, Nonlinear Analysis: Theory, Methods and Applications, 70 (13) (2009) 1246-1259.

[9] LJ. ĆIRIĆ, Some Recent Results in Metrical Fixed Point Theory, University of Belgrade, Beograd 2003, Serbia.

[10] R. P. Agarwal, E.L Karapinar, D. O'Regan, A. F. Roldan-Lopez-DeHIERRO,Fixed Point Theory in Metric Type Spaces, Springer International Publishing Switzerland 2015.

[11] W. Kirk, N. ShahZAD, Fixed Point Theory in Distance Spaces, Springer International Publishing Switzerland 2014.

[12] Suzana Aleksić, Z. Kadelburg, Zoran D. Mitrović and S. Radenović, A new survey: Cone metric spaces, Journal of the International Mathematical Virtual Institute 9 (2019), 93-121.

[13] Deepmala, A Study on Fixed Point Theorems for Nonlinear Contractions and its Applications, Ph.D. Thesis (2014), Pt. Ravishankar Shukla University, Raipur 492 010, Chhatisgarh, India.

[14] —sc L.N. Mishra, K. Jyoti, A. Rani Vandana, Fixed point theorems with digital contractions image processing, Nonlinear Sci. Lett. 9 (2) (2018), 104-115.

[15] L.N. MishrA, On existence and behavior of solutions to some nonlinear integral equations with applications, Ph.D. Thesis (2017), National Institute of Technology, Silchar 788 010, Assam, India.

[16] L.N. Mishra, S.K. Tiwari, V.N. Mishra, I.A. Khan, Unique Fixed Point Theorems for Generalized Contractive Mappings in Partial Metric Spaces, Journal of Function Spaces, Volume 2015 (2015), Article ID 960827, 8 pages.

[17] L.N. Mishra, S.K. Tiwari, V.N. Mishra; Fixed point theorems for generalized weakly Scontractive mappings in partial metric spaces, Journal of Applied Analysis and Computation, 5 (4) (2015), . 600-612.

[18] X. Liu, M. Zhou, L.N. Mishra, V.N. Mishra, B. Damuanović, Common fixed point theorem of six self-mappings in Menger spaces using $\left(C L R_{S T}\right)$ property, Open Mathematics, 2018; 16: 14231434.

[19] L.N. Mishra, H.M. SRivastava, M. Sen, On existence results for some nonlinear functional-integral equations in Banach algebra with applications, International Journal of Analysis and Applications, 11 (1) (2016), 1-10.

[20] L.N. Mishra, M. Sen, R.N. Mohapatra, On existence theorems for some generalized nonlinear functional-integral equations with applications, Filomat, 31 (7) (2017) 2081-2091.

[21] L.N. Mishra, R.P. Agarwal, On existence theorems for some nonlinear functionalintegral equations, Dynamic Systems and Applications, 25 (2016) 303-320.

[22] Deepmala And H. K. Pathak, A study on some problems on existence of solutions for nonlinear functional-integral equations, Acta Mathematica Scientia, 33 B(5) (2013), 13051313. 